\title{
Response of Ornamental Plants, Study Sites and their Interaction to Various Pollutants in Respect of APTI, API, Physiological and Biochemical Attributes
}

\author{
N. Amrutha Pavani*, M. Raja Naik, R. Nagaraju, G. Srinivasa Rao, \\ V. V. Padmaja and B. Srinivasulu
}

College of Horticulture (Dr. YSRHU), Anantharajupeta, Andhra Pradesh, India

*Corresponding author

\section{A B S T R A C T}

\begin{tabular}{l} 
K e y w o r d s \\
Horticultural \\
ornamental plants, \\
physiological and \\
biochemical \\
attributes, sites, \\
various pollutants \\
and landscaping \\
\hline Article Info \\
\hline $\begin{array}{l}\text { Accepted: } \\
\text { 04 November } 2020 \\
\text { Available Online: } \\
\text { 10 December } 2020\end{array}$ \\
\hline
\end{tabular}

The present investigation was carried out during the year 2019-2020 at four study sites. The ornamental plant i.e. OP-16 among plants, $S_{1}$ among sites and OP-16 X $S_{1}$ among interactions exhibited maximum total chlorophyll content. Relative Water Content (RWC) recorded was highest in OP-18 (91.68) among plants, $\mathrm{S}_{3}$ (81.20) among sites. OP X S interaction had no significant effect on RWC. Ascorbic acid content recorded was maximum in OP-24 (13.91 $\left.\mathrm{mg} \mathrm{g}^{-1}\right)$ among plants, $\mathrm{S}_{3}\left(4.80 \mathrm{mg} \mathrm{g}^{-1}\right)$ among sites and OP-24 $\mathrm{X} \mathrm{S}_{3}$ (14.72mg g $\mathrm{m}^{-1}$ ) among interactions. $\mathrm{pH}$ content recorded was higher in OP-23 (6.37) among species, $\mathrm{S}_{1}$ (6.31) among sites and OP X S was non significant on pH.OP-4 had

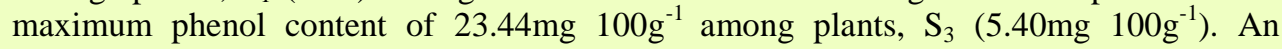
investigation for APTI revealed that OP-24 (18.82) among plants, $S_{3}$ (11.38) among study sites and OP-24 X S $\mathrm{X}_{3}$ (19.45) among interactions recorded highest APTI. With regard to Anticipated Performance Index (API), in over all, assessment study showed that OP-6, OP-19 and OP-24 are under very good performers against pollution, OP-1,OP-2, OP-3, OP-4, OP-5, OP-7, OP-8, OP-9 and OP-16 were grouped under good performers and were recommended to $S_{2}, S_{3}$ and $S_{4}$ polluted sites for green belt development and also for landscaping purpose as they mitigate and minimize air pollution load because they tend to serve as barriers and act as sink for various pollutants.

\section{Introduction}

Avenue trees, ornamental plants and shrubs are capable of removing a significant amount of air pollutants from the atmosphere and hence should be considered an integral part of any sustainable plan intended at improving air quality. Different plant species vary considerably in their susceptibility to air pollutants. Screening of plants for their sensitivity to air pollutants is of vital importance (Badamasi, 2017). The impact of air pollution on the plants can be evaluated from a combination of Air Pollution Tolerance Index (APTI) and Anticipated Performance Index (API). APTI is a tool used for the identification and classification of plants into tolerant and sensitive group based on variation in the biochemical parameters. While, the Anticipated Performance Index 
(API) value gives a good and sensible reason to classify different plant species for the development of green belt, reforestation and afforestation. Anticipated Performance Index (API) is very useful in the selection of plants that can perform dual functions of improving air quality. Increased urbanization, industrialization, mining and heavy vehicular traffic have resulted in deterioration of air quality has been a major problem from Mangampeta (rural site) to Tirupati (urban area) was the major problem since decades in Andhra Pradesh. Mangampeta (rural) in YSR Kadapa district of Andhra Pradesh is known for the emission of dust particles and different pollutants. Whereas Tirupati (urban) is well known for heavy vehicular movement and air pollution level is high and hence to find out the efficacy of available existing plant species in trapping the pollutants and dust particle delivered, the present investigation is planned.

Therefore, the aim of the present study is to determine the variation in attributes to establish the susceptibility level of different ornamental plants with reference to their tolerance and performance index which might be very useful in the selection of appropriate species which can be expected to perform well for the development of green environment and for landscaping. A very meagre or no work has been done on the development of landscaping with appropriate ornamental plants in Rayalaseema region of Andhra Pradesh particularly in above mentioned areas. Hence the present investigation was designed and planned by taking above constraints into consideration to find out the pollution tolerance level of various ornamental plants at multiple study sites to select appropriate plants which can be grown and expected to perform well for the development of greener environment along with landscaping purpose for a long way ahead.

\section{Materials and Methods}

The present investigation was carried out during the year 2019-20 at four locations viz., College of Horticulture, Anantharajupeta (Control site), Mangampeta (Barytes mining area), Rly. Kodur (Town) and Tirupati (City), Andhra Pradesh.

\section{Plant material}

Already growing, existing and commonly occurring multiple ornamental plants at four study sites were selected for investigation. College of Horticulture, Anantharajupeta (Control site- $\mathrm{S}_{1}$ ), Mangampeta (Barytes mining area and road traffic area- $\mathrm{S}_{2}$ ), Railway Kodur (Town - main road traffic area- $\mathrm{S}_{3}$ ) and Tirupati (City - main road heavy traffic area$\mathrm{S}_{4}$ ) were selected as study sites. Three plants were selected in each treatment (ornamental plant) at random and labeled properly for recording observations. Total number of plants used was 24, with three replications and two way ANOVA was used for the analysis. In all four study sites, same ornamental plant species were selected uniformly and tagged randomly as per replication and details of plant species selected are given below.

\section{Treatment (ornamental plants) details}

\begin{tabular}{|c|c|c|}
\hline S. No & Botanical name & Notation \\
\hline $\mathbf{1}$ & Azadirachta indica & OP-1 \\
\hline $\mathbf{2}$ & Bauhinia purpurea & OP-2 \\
\hline $\mathbf{3}$ & Delonix regia & OP-3 \\
\hline $\mathbf{4}$ & Peltophorum pterocarpum & OP-4 \\
\hline $\mathbf{5}$ & Polyalthia longifolia & OP-5 \\
\hline $\mathbf{6}$ & Ficus benjamina & OP-6 \\
\hline
\end{tabular}




\begin{tabular}{|r|c|c|}
\hline $\mathbf{7}$ & Conocarpus erectus & OP-7 \\
\hline $\mathbf{8}$ & Pongamia pinnata & OP-8 \\
\hline $\mathbf{9}$ & Nerium oleander & OP-9 \\
\hline $\mathbf{1 0}$ & Acalypha hispida & OP-10 \\
\hline $\mathbf{1 1}$ & Duranta repens & OP-11 \\
\hline $\mathbf{1 2}$ & Tabernaemontana divaricata & OP-12 \\
\hline $\mathbf{1 3}$ & Hibiscus rosa-sinensis & OP-13 \\
\hline $\mathbf{1 4}$ & Pandanus sanderi & OP-14 \\
\hline $\mathbf{1 5}$ & Tecoma stans & OP-15 \\
\hline $\mathbf{1 6}$ & Bougainvillea glabra & OP-16 \\
\hline $\mathbf{1 7}$ & Dieffenbachia amoena & OP-17 \\
\hline $\mathbf{1 8}$ & Sansevieria trifasciata & OP-18 \\
\hline $\mathbf{1 9}$ & Furcraea foetida & OP-19 \\
\hline $\mathbf{2 0}$ & Roystonea regia & OP-20 \\
\hline $\mathbf{2 1}$ & Wodyetia bifurcata & OP-21 \\
\hline $\mathbf{2 2}$ & Cycus cercinalis & OP-22 \\
\hline $\mathbf{2 3}$ & Catharanthus roseus & OP-23 \\
\hline $\mathbf{2 4}$ & Polianthes tuberosa & OP-24 \\
\hline
\end{tabular}

To assess the impact of air, vehicular pollution and dust particles from road sides and from control site were collected from fully matured leaves during morning hours (Akilan and Nandhakumar, 2016). The leaf samples were collected in polythene covers and were carried to the laboratory for analysis in the ice box. Leaves facing the roadside were plucked mainly during the peak days at a height of $1.5 \mathrm{~m}$ (Tsega and Deviprasad, 2014; Kaur and Nagpal, 2017).

The leaf samples were analyzed for various physiological and biochemical attributes by using standard procedures followed for total chlorophyll content (Lichthenthaler and Burchmanne, 1987), RWC (Singh (1977), ascorbic acid content (Ranganna, 1977), leaf pH (Prasad and Rao (1982), phenol content (Singleton et al., 1965), proline content (Bates et al., 1973), APTI and API (Govindaraju et al., 2012).

Anticipated Performance Index (API) of plant species

After calculating the APTI, the Anticipated Performance Index (API) is presented for various ornamental species as follows based on their response to pollution.
Rating used for anticipated performance index of plant species (Govindaraju et al., 2012) (Table 2). Variation of different physiological, biochemical parameters, pattern and their significance level were computed using Analysis of Variance Technique (Two-factor analysis) with ornamental plants and different sites as two factors for analysis. The significance of the analyzed data was tabled at 5 per cent level of significance.

\section{Results and Discussion}

\section{Total chlorophyll content}

The study revealed that total chlorophyll content in all the ornamental plants varied with the pollution status of the study area. The ornamental plant OP-16 contained maximum total chlorophyll $\left(3.90 \mathrm{mg} \mathrm{g}^{-1}\right)$ which was followed by OP-24 (1.96 mg g $\left.{ }^{-1}\right)$. Site $\mathrm{S}_{1}$ showed maximum total chlorophyll $(1.37 \mathrm{mg}$ $\mathrm{g}^{-1}$ ) among different sites which was followed by $\mathrm{S}_{4}\left(1.31 \mathrm{mg} \mathrm{g}^{-1}\right), \mathrm{S}_{2}\left(1.04 \mathrm{mg} \mathrm{g}^{-1}\right)$ and $\mathrm{S}_{3}$ $\left(0.96 \mathrm{mg} \mathrm{g}^{-1}\right)$. Among interactions, OP-16 X $\mathrm{S}_{4}$ had significantly highest total chl. (4.62 mg $\mathrm{g}^{-1}$ ) followed by OP-16 X $\mathrm{S}_{1}\left(4.41 \mathrm{mg} \mathrm{g}^{-1}\right)$.

The results obtained showed that among ornamental plants, Bougainvillea glabra (OP- 
16) at control site $\left(S_{1}\right.$-College of Horticulture, Anantharajupeta) exhibited highest total chlorophyll content. Plants having higher chlorophyll content are able to produce more amounts of carbohydrates by increase in the rate of photosynthesis even under polluted environments. The higher the levels of pollutants, the lower the chlorophyll content as certain pollutants in totality reduce the total chlorophyll content was observed at $\mathrm{S}_{2}, \mathrm{~S}_{3}$ and $\mathrm{S}_{4}$. Reduction in chlorophyll content brought by acidic pollutants like $\mathrm{SO}_{2}$ which causes phaeophytin formation by acidification of chlorophyll due to $\mathrm{NO}_{2}, \mathrm{SO}_{2}$ and $\mathrm{O}_{3}$ exposure have also been reported by (Gharge and Menon, 2012; Rai et al., 2013).

\section{Relative water content}

A perusal of the data revealed that, ornamental plants studied showed maximum RWCin OP-18 (91.68\%) followed by OP-19 $(88.54 \%)$ and this was on par with OP-17 $(87.80 \%)$. Significantly highest RWC of 81.20 per cent was observed at $S_{3}$ followed by $\mathrm{S}_{2}(77.75 \%), \mathrm{S}_{4}(73.88 \%)$. The interaction effect of ornamental plants $\mathrm{x}$ sites was not showed significant response on the RWC. The highest value of RWC in Sansevieria trifasciata (OP-18) and Furcraea foetida (OP19) may be ascribed due to its higher resistance capacity to stress conditions. The plant with high RWC under polluted conditions were tolerant to pollutants (Chandawat et al., 2011; Soni et al., 2017). RWC of polluted area $\left(S_{2}, S_{3}, S_{4}\right)$ was higher than those from the control plants $\left(S_{1}\right)$. Maximum value of RWC at polluted site further provide evidence that plants retain more water than those at less polluted site this might be due to adaptive feature which helps in maintaining its physiological balance against pollution stress which may be tolerant to pollutants. High relative water content contributes to the normal functioning of the biological processes (Meerabai et al., 2012) and favors drought and pollution resistant in plants.

\section{Ascorbic acid}

A significant variation was noticed in the leaf ascorbic acid content of ornamental plant species, sites and their interaction. Among 24 ornamental plants studied, significantly superior value for ascorbic acid content was recorded in OP-24 (13.91 $\left.\mathrm{mg} \mathrm{g}^{-1}\right)$ which was followed by OP-19 (10.47mg g $\left.\mathrm{g}^{-1}\right)$, OP-23 (6.90 $\left.\mathrm{mg} \mathrm{g}^{-1}\right)$. Among 4 sites, significantly higher ascorbic acid content $\left(4.80 \mathrm{mg} \mathrm{g}^{-1}\right)$ was recorded in $S_{3}$ followed by $S_{2}\left(4.10 \mathrm{mg} \mathrm{g}^{-1}\right)$, $\mathrm{S}_{4}\left(3.31 \mathrm{mg} \mathrm{g}^{-1}\right)$ and $\mathrm{S}_{1}\left(2.60 \mathrm{mg} \mathrm{g}^{-1}\right)$. Among combination of interaction treatments, OP-24 $\mathrm{X} \mathrm{S}_{3}$ registered significantly highest ascorbic acid content $\left(14.72 \mathrm{mg} \mathrm{g}^{-1}\right)$ followed by OP$24 \mathrm{X} \mathrm{S}_{2}\left(14.13 \mathrm{mg} \mathrm{g}^{-1}\right)$. Ascorbic acid is an antioxidant that increases the resistance of plants against air pollutants. Among multiple polluted sites, Polianthes tuberosa (OP-24) grown at $\mathrm{S}_{3}$ (Railway Kodur town, highly polluted area) recorded highest ascorbic acid content. Plant species with high amount of ascorbic acid are considered to be tolerant to air pollutants (Deepalakshmi et al., 2013; Kousar et al., 2014).

The increase in level of ascorbic acid may be due to the defense mechanism of the species against pollution load at different sites. Similarly, Bhattacharya et al., (2012) also recorded higher values of ascorbic acid in plant leaves at polluted sites. The ascorbic acid content from the plants in the polluted sites $\left(S_{2}\right.$ and $\left.S_{3}\right)$ was higher than that from the control site plants $\left(S_{1}\right)$, in this present study, this agrees with the reports of Chandawat et al., (2011), Meerabai et al., (2012) and Rai et al., (2013) who found higher levels of ascorbic acid in the leaves of most tolerant plants and those at the polluted sites and this suggests their tolerance to the air pollutants. 


\section{Leaf pH}

The data made available in table 3 represents that significantly maximum leaf $\mathrm{pH}$ content of 6.37 was noticed in OP-23 which was on par with OP-6 (6.26) and OP-7 (6.18). Among the sites, $\mathrm{S}_{4}$ (6.31) recorded significantly maximum $\mathrm{pH}$ followed by $\mathrm{S}_{1}(6.15)$ and $\mathrm{S}_{2}$ (5.98) and most acidic $\mathrm{pH}$ was recorded at $\mathrm{S}_{3}$ (5.67). The $\mathrm{pH}$ was not significantly influenced by combination of ornamental plants x sites.

Leaf $\mathrm{pH}$ is the $\mathrm{pH}$ of the concentrates from the leaves of the plant. It was observed that among ornamental plants investigated for $\mathrm{pH}$, Catharanthus roseus (OP-23) grown at control site $\left(S_{1}\right)$ exhibited maximum $\mathrm{pH}$ content. All the plant samples collected from polluted site $\left(\mathrm{S}_{2}, \mathrm{~S}_{3}\right.$ and $\left.\mathrm{S}_{4}\right)$ exhibited $\mathrm{pH}$ towards acidic side which may be due to the presence of $\mathrm{CO}_{2}, \mathrm{SO}_{2}$ and $\mathrm{NO}_{\mathrm{x}}$ in the ambient air causing a change in $\mathrm{pH}$ of the leaf sap towards acidic site (Das and Prasad, 2010).Plants from the polluted site had a $\mathrm{pH}$ towards acidic side, whereas those from the control site showed neutral to slightly alkaline range (Rai et al., 2013; Bora and Joshi, 2014).The results obtained showed that $\mathrm{pH}$ of leaf extract was acidic, which may be due to diffusion of gaseous air pollutants like $\mathrm{NO}_{2}$, $\mathrm{CO}_{2}$ and $\mathrm{SO}_{2}$ in the plant cell sap and when plants are suffering from air pollutants (especially $\mathrm{SO}_{2}$ ) as their cellular fluid would produce massive $\mathrm{H}^{+}$to react with $\mathrm{SO}_{2}$, which enters through stomata and intercellular space from air, so that $\mathrm{H}_{2} \mathrm{SO}_{4}$ is generated and then leaf $\mathrm{pH}$ reduces. The results obtained from the present investigation are in agreement with the findings of Ghassanen et al., (2016), Kaur and Nagpal (2017) (Table 4).

\section{Phenol content}

Phenol content varied significantly due to the influence of ornamental plants, locations and their interactions. Among the ornamental plants studied, significantly superior value for phenol content was recorded in OP-4 (23.44 mg $100 \mathrm{~g}^{-1}$ ) followed by OP-3 (18.53 mg 100 $\mathrm{g}^{-1}$ ), OP-7 (10.06 mg $\left.100 \mathrm{~g}^{-1}\right)$. Among sites, $\mathrm{S}_{3}$ had highest phenol content $(5.40 \mathrm{mg} 100$ $\left.\mathrm{g}^{-1}\right)$ followed by $S_{2}\left(4.81 \mathrm{mg} 100 \mathrm{~g}^{-1}\right), \mathrm{S}_{4}(4.23$ $\left.\mathrm{mg} 100 \mathrm{~g}^{-1}\right)$ and $\mathrm{S}_{1}\left(3.72 \mathrm{mg} 100 \mathrm{~g}^{-1}\right)$. The interaction of ornamental plants $\mathrm{x}$ multiple locations did not show significant influence on phenol content.

Phenols act as a free radical scavengers to protect plants away from damage by oxidative stress. They play important role in maintaining cellular osmotic potential when plant is exposed to stress conditions especially air pollution. The highest concentration of total phenols in the leaves of Peltophorum pterocarpum (OP-4) may be attributed to its genetic constitution and moreover, due to its highest tolerance to the pollution as an adaptation to avoid the adverse effect of air pollution at $\mathrm{S}_{3}$ (Railway Kodur). The increase in the concentration of total phenols in the plants growing at $S_{3}$ may be ascribed to higher pollution load near to the location particularly with vehicles and other pollutants. The results are in agreement with the findings of Chandawat et al., (2014), Mohsenpour et al., (2015) who studied the increase in phenolic compounds in plants exposed to various pollutants.

\section{Air Pollution Tolerance Index (APTI)}

Data furnished revealed that OP-24 was found significantly superior in recording higher APTI value (18.82) followed by OP-19 (15.82) and OP-23 (12.42). Among 4 sites, $\mathrm{S}_{3}$ exerted highest value (11.38) followed by $S_{2}$ (10.75), $S_{4}$ (9.99) and lowest at $S_{1}$ (9.01). In combinations, OP-24 $\mathrm{X} \quad \mathrm{S}_{3}$ showed significantly highest APTI (19.45) which was on par with OP-24 X S 2 (19.25) and OP-24 X $\mathrm{S}_{4}$ (18.65) (Table 5). 
Table.1 Gradation of plant species based on Air Pollution Tolerance Index as well as morphological parameters and socio-economic importance

\begin{tabular}{|c|c|c|c|}
\hline \multicolumn{2}{|l|}{ Grading Character } & Pattern of assessment & Grade allotted \\
\hline \multirow[t]{5}{*}{ Tolerance } & \multirow{5}{*}{$\begin{array}{l}\text { APTI } \\
\text { (Tolerance) }\end{array}$} & $9.0-12.0$ & + \\
\hline & & $12.1-15.0$ & ++ \\
\hline & & $15.1-18.0$ & +++ \\
\hline & & $18.1-21.0$ & ++++ \\
\hline & & $21.1-24.0$ & +++++ \\
\hline \multirow{8}{*}{$\begin{array}{l}\text { Biological and socio - } \\
\text { economic }\end{array}$} & \multirow[t]{3}{*}{ Plant habit } & Small & - \\
\hline & & Medium & + \\
\hline & & Large & ++ \\
\hline & \multirow{3}{*}{ Canopy structure } & Sparse/irregular/globular & - \\
\hline & & $\begin{array}{l}\text { Spreading crown/open/semi- } \\
\text { dense } \\
\text { crown/open/semi-dense }\end{array}$ & + \\
\hline & & Spreading dense & ++ \\
\hline & \multirow[t]{2}{*}{ Type of plant } & Deciduous & - \\
\hline & & Evergreen & + \\
\hline \multirow[t]{10}{*}{ Laminar structure } & \multirow[t]{3}{*}{ Leaf size } & Small & - \\
\hline & & Medium & + \\
\hline & & Large & ++ \\
\hline & \multirow[t]{2}{*}{ Texture } & Smooth & - \\
\hline & & Coriaceous & + \\
\hline & \multirow[t]{2}{*}{ Hardiness } & Delineate & - \\
\hline & & Hardy & + \\
\hline & \multirow[t]{3}{*}{ Economic value } & Less than three uses & - \\
\hline & & Three or four uses & + \\
\hline & & Five or more uses & ++ \\
\hline
\end{tabular}

Maximum grades scored by any plant can be $=16$

Source: (Govindaraju et al., 2012)

Table.2 Rating used for anticipated performance index of plant species

(Govindaraju et al., 2012)

\begin{tabular}{|c|c|l|}
\hline Grade & Score (\%) & Assessment category \\
\hline $\mathbf{0}$ & Up to 30 & Not recommended \\
\hline $\mathbf{1}$ & $31-40$ & Very poor \\
\hline $\mathbf{2}$ & $41-50$ & Poor \\
\hline $\mathbf{3}$ & $51-60$ & Moderate \\
\hline $\mathbf{4}$ & $61-70$ & Good \\
\hline $\mathbf{5}$ & $71-80$ & Very good \\
\hline $\mathbf{6}$ & $81-90$ & Excellent \\
\hline $\mathbf{7}$ & $91-100$ & Best \\
\hline
\end{tabular}


Table.3 Response of ornamental plants (OP), sites (S) and their interaction to various pollutants in respect of, total chl, RWC, ascorbic acid

\begin{tabular}{|c|c|c|c|c|c|c|c|c|c|c|c|c|c|c|c|}
\hline \multirow{2}{*}{$\begin{array}{c}\text { Ornamental } \\
\text { plant }\end{array}$} & \multicolumn{5}{|c|}{ Total chlorophyll (mg g $\left.\mathbf{g}^{-1}\right)$} & \multicolumn{5}{|c|}{ RWC (\%) } & \multicolumn{5}{|c|}{ Ascorbic acid (mg g-1) } \\
\hline & $\mathrm{S}_{1}$ & $\mathrm{~S}_{2}$ & $\mathrm{~S}_{3}$ & $\mathrm{~S}_{4}$ & Mean & 63.81 & 75.65 & 76.54 & 72.63 & 72.16 & $\mathrm{~S}_{1}$ & $\mathrm{~S}_{2}$ & $\mathrm{~S}_{3}$ & $\mathrm{~S}_{4}$ & Mean \\
\hline OP-1 & 1.40 & 1.29 & 1.10 & 1.29 & 1.27 & 48.35 & 70.18 & 75.16 & 64.85 & 64.64 & 4.16 & 5.33 & 5.92 & 4.74 & 5.04 \\
\hline OP-2 & 2.08 & 1.42 & 1.28 & 1.53 & 1.58 & 44.85 & 55.42 & 57.38 & 48.18 & 51.46 & 2.60 & 3.72 & 4.30 & 3.13 & 3.44 \\
\hline OP-3 & 1.39 & 1.16 & 1.05 & 1.34 & 1.23 & 57.94 & 70.74 & 77.12 & 64.90 & 67.68 & 5.69 & 6.74 & 7.32 & 6.15 & 6.48 \\
\hline OP-4 & 1.86 & 1.56 & 1.47 & 1.81 & 1.67 & 75.66 & 77.66 & 84.43 & 75.57 & 78.33 & 5.00 & 6.06 & 6.65 & 5.48 & 5.80 \\
\hline OP-5 & 0.90 & 0.59 & 0.47 & 0.72 & 0.67 & 81.43 & 84.82 & 87.69 & 82.49 & 84.11 & 1.19 & 4.43 & 5.32 & 3.84 & 3.70 \\
\hline OP-6 & 1.05 & 0.92 & 0.84 & 1.06 & 0.97 & 50.82 & 53.38 & 58.35 & 50.67 & 53.30 & 1.56 & 2.70 & 3.29 & 2.11 & 2.42 \\
\hline OP-7 & 1.17 & 0.98 & 1.04 & 1.28 & 1.12 & 74.64 & 84.08 & 86.60 & 81.09 & 81.60 & 0.82 & 1.97 & 2.56 & 1.38 & 1.68 \\
\hline OP-8 & 1.62 & 1.22 & 1.17 & 1.50 & 1.38 & 67.73 & 76.94 & 79.53 & 71.17 & 73.84 & 1.25 & 5.26 & 5.33 & 3.24 & 3.77 \\
\hline OP-9 & 1.57 & 1.00 & 0.95 & 1.45 & 1.24 & 66.85 & 75.52 & 77.66 & 68.90 & 72.24 & 1.22 & 5.59 & 8.57 & 2.08 & 4.37 \\
\hline OP-10 & 1.37 & 0.99 & 0.93 & 1.27 & 1.14 & 78.16 & 83.46 & 84.65 & 79.51 & 81.45 & 2.63 & 3.74 & 4.33 & 3.16 & 3.47 \\
\hline OP-11 & 1.10 & 0.87 & 0.81 & 1.11 & 0.98 & 64.84 & 72.12 & 76.82 & 68.23 & 70.50 & 0.31 & 1.48 & 2.06 & 0.89 & 1.19 \\
\hline OP-12 & 1.07 & 0.72 & 0.66 & 1.01 & 0.86 & 77.51 & 83.37 & 83.46 & 78.47 & 80.70 & 0.98 & 2.53 & 3.12 & 1.54 & 2.05 \\
\hline OP-13 & 0.90 & 0.66 & 0.60 & 0.89 & 0.76 & 54.08 & 66.76 & 73.31 & 61.43 & 63.90 & 0.50 & 1.66 & 2.25 & 1.08 & 1.37 \\
\hline OP-14 & 0.99 & 0.72 & 0.65 & 0.99 & 0.84 & 77.31 & 84.54 & 89.25 & 82.80 & 83.48 & 0.37 & 1.54 & 2.13 & 0.95 & 1.25 \\
\hline OP-15 & 1.03 & 0.80 & 0.73 & 1.10 & 0.92 & 75.76 & 83.37 & 85.45 & 78.11 & 80.67 & 0.31 & 2.07 & 3.13 & 1.49 & 1.75 \\
\hline OP-16 & 4.41 & 3.31 & 3.24 & 4.62 & 3.90 & 84.42 & 87.65 & 89.94 & 89.20 & 87.80 & 0.82 & 1.97 & 2.56 & 1.38 & 1.68 \\
\hline OP-17 & 0.94 & 0.75 & 0.65 & 0.98 & 0.83 & 89.85 & 92.59 & 94.53 & 89.75 & 91.68 & 0.65 & 1.81 & 2.40 & 1.22 & 1.52 \\
\hline OP-18 & 0.75 & 0.47 & 0.41 & 0.69 & 0.58 & 86.36 & 89.34 & 92.18 & 86.30 & 88.54 & 1.87 & 3.01 & 3.59 & 2.42 & 2.72 \\
\hline OP-19 & 0.87 & 0.71 & 0.58 & 0.90 & 0.77 & 76.89 & 82.00 & 92.58 & 79.87 & 82.84 & 9.75 & 10.71 & 11.30 & 10.12 & 10.47 \\
\hline OP-20 & 0.98 & 0.75 & 0.69 & 1.00 & 0.86 & 79.43 & 87.72 & 89.44 & 83.94 & 85.13 & 0.37 & 1.54 & 2.13 & 0.95 & 1.25 \\
\hline OP-21 & 1.42 & 1.16 & 1.07 & 1.34 & 1.25 & 72.17 & 73.31 & 75.55 & 72.63 & 73.42 & 0.56 & 1.72 & 2.31 & 1.14 & 1.43 \\
\hline OP-22 & 0.60 & 0.32 & 0.26 & 0.50 & 0.42 & 67.69 & 75.55 & 80.04 & 70.29 & 73.39 & 0.31 & 1.48 & 2.06 & 0.89 & 1.19 \\
\hline OP-23 & 1.16 & 0.78 & 0.68 & 1.05 & 0.92 & 67.81 & 79.90 & 81.13 & 72.14 & 75.25 & 6.12 & 7.16 & 7.75 & 6.58 & 6.90 \\
\hline OP-24 & 2.32 & 1.77 & 1.70 & 2.04 & 1.96 & 70.18 & $\mathbf{7 7 . 7 5}$ & 81.20 & 73.88 & & 13.25 & 14.13 & 14.72 & 13.54 & 13.91 \\
\hline Mean & 1.37 & 1.04 & 0.96 & 1.31 & & OP & S & OP $x \mathrm{~S}$ & & & 2.60 & 4.10 & 4.80 & 3.31 & \\
\hline \multirow[b]{2}{*}{$\mathrm{CD}[P=0.05]$} & OP & $\mathrm{S}$ & OP $x \mathrm{~S}$ & & & 3.13 & 1.27 & NS & & & OP & $\mathrm{S}$ & OP $\times \mathrm{S}$ & & \\
\hline & 0.04 & 0.02 & 0.09 & & & 63.81 & 75.65 & 76.54 & 72.63 & 72.16 & 0.21 & 0.08 & 0.42 & & \\
\hline
\end{tabular}


Table.4 Response of ornamental plants (OP), sites (S) and their interaction to various pollutants in respect of $\mathrm{pH}$, phenol content and APTI

\begin{tabular}{|c|c|c|c|c|c|c|c|c|c|c|c|c|c|c|c|}
\hline \multirow{2}{*}{$\begin{array}{c}\text { Ornamental } \\
\text { plant }\end{array}$} & \multicolumn{5}{|c|}{ pH } & \multicolumn{5}{|c|}{ Phenol content $\left(\mu \mathrm{g} \mathrm{g}^{-1} \mathrm{FW}\right)$} & \multicolumn{5}{|c|}{ APTI } \\
\hline & $\mathrm{S}_{1}$ & $\mathrm{~S}_{2}$ & $\mathrm{~S}_{3}$ & $\mathrm{~S}_{4}$ & Mean & $\mathrm{S}_{1}$ & $\mathrm{~S}_{2}$ & $\mathrm{~S}_{3}$ & $\mathrm{~S}_{4}$ & Mean & $\mathrm{S}_{1}$ & $\mathrm{~S}_{2}$ & $\mathrm{~S}_{3}$ & $\mathrm{~S}_{4}$ & Mean \\
\hline OP-1 & 5.97 & 6.17 & 5.97 & 6.27 & 6.09 & 4.81 & 5.99 & 6.57 & 5.40 & 5.69 & 9.50 & 11.61 & 11.92 & 10.91 & 10.98 \\
\hline OP-2 & 6.17 & 5.87 & 6.07 & 6.27 & 6.09 & 5.33 & 6.38 & 6.97 & 5.79 & 6.12 & 6.97 & 9.77 & 10.73 & 8.96 & 9.11 \\
\hline OP-3 & 6.17 & 5.97 & 5.67 & 6.57 & 6.09 & 17.95 & 18.73 & 19.31 & 18.14 & 18.53 & 8.76 & 10.43 & 10.75 & 9.76 & 9.93 \\
\hline OP-4 & 6.17 & 6.07 & 5.77 & 6.37 & 6.09 & 22.95 & 23.61 & 24.20 & 23.02 & 23.44 & 9.78 & 11.78 & 12.61 & 11.04 & 11.30 \\
\hline OP-5 & 6.27 & 6.17 & 5.27 & 6.47 & 6.04 & 7.19 & 8.20 & 8.79 & 7.62 & 7.95 & 8.41 & 10.82 & 11.57 & 10.37 & 10.29 \\
\hline OP-6 & 6.57 & 6.17 & 5.67 & 6.63 & 6.26 & 1.63 & 2.76 & 3.35 & 2.18 & 2.48 & 9.32 & 10.43 & 10.95 & 9.91 & 10.15 \\
\hline OP-7 & 6.17 & 6.07 & 5.77 & 6.73 & 6.18 & 9.33 & 10.30 & 10.88 & 9.71 & 10.06 & 5.68 & 6.75 & 7.61 & 6.20 & 6.56 \\
\hline OP-8 & 6.27 & 5.97 & 5.37 & 6.17 & 5.94 & 6.76 & 7.79 & 8.37 & 7.20 & 7.53 & 8.44 & 12.26 & 12.22 & 10.63 & 10.89 \\
\hline OP-9 & 6.47 & 6.07 & 5.47 & 6.27 & 6.07 & 0.53 & 1.69 & 2.28 & 1.11 & 1.40 & 7.75 & 11.72 & 13.56 & 8.75 & 10.45 \\
\hline OP-10 & 6.37 & 6.27 & 6.07 & 6.57 & 6.32 & 3.11 & 4.21 & 4.80 & 3.62 & 3.93 & 8.71 & 10.32 & 10.85 & 9.41 & 9.82 \\
\hline OP-11 & 6.27 & 6.37 & 5.97 & 6.47 & 6.27 & 0.44 & 1.60 & 2.19 & 1.01 & 1.31 & 8.05 & 9.44 & 9.70 & 8.64 & 8.95 \\
\hline OP-12 & 6.07 & 5.97 & 5.67 & 6.17 & 5.97 & 0.85 & 2.00 & 2.59 & 1.42 & 1.72 & 7.18 & 8.94 & 9.70 & 7.95 & 8.44 \\
\hline OP-13 & 6.07 & 5.97 & 5.87 & 6.17 & 6.02 & 0.26 & 1.42 & 2.01 & 0.84 & 1.13 & 8.10 & 9.46 & 9.83 & 8.62 & 9.00 \\
\hline OP-14 & 6.27 & 6.07 & 5.67 & 6.47 & 6.12 & 0.51 & 1.67 & 2.26 & 1.09 & 1.38 & 5.68 & 7.74 & 8.70 & 6.87 & 7.25 \\
\hline OP-15 & 5.67 & 5.47 & 4.90 & 6.07 & 5.53 & 0.79 & 1.94 & 2.53 & 1.35 & 1.65 & 7.94 & 9.78 & 10.72 & 9.37 & 9.45 \\
\hline OP-16 & 6.07 & 5.87 & 5.87 & 6.27 & 6.02 & 1.06 & 2.21 & 2.80 & 1.63 & 1.93 & 8.42 & 10.17 & 10.90 & 9.33 & 9.71 \\
\hline OP-17 & 5.97 & 5.87 & 5.67 & 6.07 & 5.89 & 0.62 & 1.78 & 2.37 & 1.19 & 1.49 & 8.89 & 9.99 & 10.54 & 9.80 & 9.80 \\
\hline OP-18 & 5.97 & 5.77 & 5.37 & 5.97 & 5.77 & 0.11 & 1.29 & 1.87 & 0.70 & 0.99 & 10.24 & 11.17 & 11.58 & 10.62 & 10.90 \\
\hline OP-19 & 6.07 & 5.67 & 5.27 & 6.27 & 5.82 & 0.99 & 2.15 & 2.73 & 1.56 & 1.86 & 15.38 & 15.91 & 15.97 & 16.02 & 15.82 \\
\hline OP-20 & 6.07 & 5.67 & 5.00 & 6.17 & 5.73 & 1.13 & 2.27 & 2.86 & 1.69 & 1.99 & 7.95 & 9.21 & 10.49 & 8.68 & 9.08 \\
\hline OP-21 & 5.97 & 5.77 & 5.67 & 6.07 & 5.87 & 1.03 & 2.19 & 2.77 & 1.60 & 1.90 & 8.36 & 9.99 & 10.53 & 9.25 & 9.53 \\
\hline OP-22 & 6.07 & 5.97 & 5.97 & 6.17 & 6.04 & 0.51 & 1.67 & 2.25 & 1.08 & 1.38 & 7.42 & 8.28 & 8.87 & 7.87 & 8.11 \\
\hline OP-23 & 6.37 & 6.37 & 6.27 & 6.47 & 6.37 & 0.84 & 1.99 & 2.58 & 1.41 & 1.70 & 11.36 & 12.77 & 13.49 & 12.06 & 12.42 \\
\hline OP-24 & 6.17 & 6.07 & 5.87 & 6.27 & 6.09 & 0.48 & 1.64 & 2.23 & 1.06 & 1.35 & 17.95 & 19.25 & 19.45 & 18.65 & 18.82 \\
\hline Mean & 6.15 & 5.98 & 5.67 & 6.31 & & 3.72 & 4.81 & 5.40 & 4.23 & & 9.01 & 10.75 & 11.38 & 9.99 & \\
\hline \multirow{3}{*}{$\mathrm{CD}[P=0.05]$} & $\mathrm{OP}$ & $\mathrm{S}$ & OP xS & & & $\mathrm{OP}$ & $\mathrm{S}$ & OP xS & & & $\mathrm{OP}$ & $\mathrm{S}$ & $\mathrm{OP} \times \mathrm{S}$ & & \\
\hline & 0.23 & 0.09 & NS & & & 0.31 & 0.12 & NS & & & 0.42 & 0.17 & 0.85 & & \\
\hline & & & & & & 4.81 & 5.99 & 6.57 & 5.40 & 5.69 & 9.50 & 11.61 & 11.92 & 10.91 & 10.98 \\
\hline
\end{tabular}


Table.4 Gradation of plant species based on air pollution tolerance index (APTI) as well as morphological parameters and socioeconomic importance

\begin{tabular}{|c|c|c|c|c|c|c|c|c|c|c|c|c|}
\hline $\begin{array}{c}\text { Ornamental } \\
\text { plant }\end{array}$ & APTI & $\begin{array}{l}\text { Plant } \\
\text { habit }\end{array}$ & $\begin{array}{c}\text { Canopy } \\
\text { structure }\end{array}$ & $\begin{array}{c}\text { Type of } \\
\text { plant }\end{array}$ & $\begin{array}{c}\text { Leaf } \\
\text { size }\end{array}$ & $\begin{array}{c}\text { Leaf } \\
\text { texture }\end{array}$ & Hardiness & $\begin{array}{c}\text { Economic } \\
\text { value }\end{array}$ & $\begin{array}{l}\text { Total } \\
\text { Plus }\end{array}$ & $\begin{array}{c}\% \\
\text { Score }\end{array}$ & $\begin{array}{c}\text { API } \\
\text { Grade }\end{array}$ & $\begin{array}{c}\text { Assessment } \\
\text { category }\end{array}$ \\
\hline OP-1 & ++ & ++ & ++ & - & + & + & + & ++ & 11 & 68.75 & 4 & Good \\
\hline OP-2 & ++ & ++ & + & - & ++ & + & + & + & 10 & 62.5 & 4 & Good \\
\hline OP-3 & ++ & ++ & ++ & + & ++ & - & + & - & 10 & 62.5 & 4 & Good \\
\hline OP-4 & ++ & ++ & ++ & + & ++ & - & + & + & 11 & 68.75 & 4 & Good \\
\hline OP-5 & ++ & + & ++ & + & ++ & + & + & + & 11 & 68.75 & 4 & Good \\
\hline OP-6 & ++ & ++ & ++ & + & + & + & + & ++ & 12 & 75 & 5 & Very good \\
\hline OP-7 & + & ++ & ++ & + & + & + & + & ++ & 11 & 68.75 & 4 & Good \\
\hline OP-8 & ++ & ++ & ++ & - & + & - & + & ++ & 10 & 62.5 & 4 & Good \\
\hline OP-9 & ++ & + & + & + & ++ & + & + & + & 10 & 62.5 & 4 & Good \\
\hline OP-10 & ++ & + & + & + & + & + & - & - & 7 & 43.75 & 2 & Poor \\
\hline OP-11 & + & + & + & + & - & - & + & - & 5 & 31.25 & 1 & Very poor \\
\hline OP-12 & + & + & + & + & + & - & + & + & 7 & 43.75 & 2 & Poor \\
\hline OP-13 & + & + & + & + & + & - & + & + & 7 & 43.75 & 2 & Poor \\
\hline OP-14 & + & - & + & + & ++ & + & + & - & 7 & 43.75 & 2 & Poor \\
\hline OP-15 & ++ & + & ++ & + & + & - & + & - & 8 & 50 & 2 & Poor \\
\hline OP-16 & ++ & ++ & ++ & + & + & - & + & + & 10 & 62.5 & 4 & Good \\
\hline OP-17 & + & - & - & + & ++ & + & + & - & 6 & 37.5 & 1 & Very poor \\
\hline OP-18 & ++ & - & - & + & ++ & + & + & + & 8 & 50 & 2 & Poor \\
\hline OP-19 & +++ & + & ++ & + & ++ & + & + & + & 12 & 75 & 5 & Very good \\
\hline OP-20 & + & + & - & + & ++ & + & + & - & 7 & 43.75 & 2 & Poor \\
\hline OP-21 & + & + & - & + & ++ & + & + & - & 7 & 43.75 & 2 & Poor \\
\hline OP-22 & + & + & - & + & ++ & + & + & - & 7 & 43.75 & 2 & Poor \\
\hline OP-23 & ++ & + & + & + & + & - & + & ++ & 9 & 56.25 & 3 & Moderate \\
\hline OP-24 & ++++ & + & + & + & ++ & - & + & ++ & 12 & 75 & 5 & Very good \\
\hline
\end{tabular}


Air pollution tolerance index (APTI) study proves significant in determining the tolerant and sensitive nature of plant species in environment. The results presented revealed that APTI value recorded was highest in Polianthes tuberosa (OP-24) grown at highly polluted site $\left(\mathrm{S}_{3}\right.$-Railway Kodur town). The results demonstrate that different plant species respond in different ways to air pollution and the same plant species growing in different environments may respond differently, depending on the level of air pollution in the habitat. In the present work, the variation in the APTI values could be attributed to the different responses of the plants to the four physiological factors, namely, ascorbic acid, total chlorophyll, leaf $\mathrm{pH}$ and the RWC of the leaf. These physiological factors, in turn, are affected by variation in the level of air pollution in the environment (Nwadinigwe, 2014).

Among ornamental plants investigated for APTI, Polianthes tuberosa (OP-24), Furcraea foetida (OP-19), Catharanthus roseus (OP23), Peltophorum pterocarpum (OP-4) and Azadirachta indica (OP-1) recorded highest values for APTI at polluted sites $\left(\mathrm{S}_{3}\right.$-Railway Kodur Town-highly polluted area and $\mathrm{S}_{2-}$ Mangampeta Barytes mining and road traffic area). Hence in the same corollary, our results are in clear agreement with the findings of Chaudhary and Banerjee (2009), they reported that the species having APTI value < 10 is considered as 'sensitive'; value within $10-16$ is considered as 'intermediate' and $>17$ is 'tolerant'.

The plants with high APTI values are tolerant to air pollution and can be utilized as filters/sinks to mitigate air pollution, while plants with low APTI can indicate the sensitive nature of plants which can be used as bio-indicators (Madan and Chauhan, 2015).These results are in corroboration with the findings of Jyothi and Jaya (2010) who have pointed that higher APTI values are associated with higher tolerance of plant species to air pollutants (Noor et al., 2015).

Plants with high APTI (tolerant plants) can trap and contain dust particles or smog, absorb pollutants, heat, and other gaseous emissions and improve the ambient air quality. Such plant species should be grown in polluted cities, along roads, towns, cities and in and around industrial area and also for landscaping purpose to create a sort of "curtain" that will absorb pollutants and screens the environment from their harmful effects.

\section{Anticipated performance Index (API)}

Evaluation of API and assessment of different plants based on their API values revealed that among the selected ornamental plants, Ficus benjamina (OP-6), Furcraea foetida (OP-19) and Polianthes tuberosa (OP-24) are came under the assessment category of very good performers with highest API was in the very good category with API grade 5.The highest value of API of Ficus benjamina (OP-6), Furcraea foetida (OP-19) and Polianthes tuberosa (OP-24) followed by OP-1, OP-2, OP-3, OP-4, OP-5, OP-7, OP-8, OP-9 and OP-16 (good category) may be due to their high APTI. Further, the better laminar characteristics viz., larger size, texture along with the high economic value has also enhanced it API value towards the very good and good category. Whereas the lower APTI, small leaf size and comparatively less economic importance has decreased the API value of OP-10, OP-11 making it to fall in not recommended category. The results are in accordance with Govindaraju et al., (2012), Bora and Joshi (2014), Kaur and Nagpal (2017) who have also reported more value of API for the species with higher APTI and better tree and leaf characteristics (Table 1). 
Plantation of OP-6, OP-19 and OP-24 (very good performers) followed by OP-1, OP-2, OP-3, OP-4, OP-5, OP-7, OP-8, OP-9 and OP-16 (good performers) were evaluated as first among all the plants and were a keystone species. A keystone species is one that has a disproportionate effect on its environment relative to its abundance. An ecosystem may experience a dramatic shift if a keystone species is removed, even though that species was a small part of the ecosystem by measures of biomass or productivity (Prajapati and Tripathi, 2008).

In conclusion the environmental point of view, plantation of Ficus benjamina (OP-6), Furcraea foetida (OP-19) and Polianthes tuberosa (OP-24) (very good performers as per APTI and API assessment) followed by Azadirachta indica (OP-1), Bauhinia purpurea (OP-2), Delonix regia (OP-3), Peltophorum pterocarpum (OP-4), Polyalthia longifolia (OP-5), Conocarpus erectus (OP7), Pongamia pinnata (OP-8), Nerium oleander (OP-9) and Bougainvillea glabra (OP-16) were evaluated as good performers (as per APTI and API assessment) among all the ornamental plants. Therefore the species come under very good and good category as per API assessment were the highly suitable species for plantation in and around the Mangampeta (Barytes mining and road traffic area- $\mathrm{S}_{2}$ ) and Railway Kodur (Town - main traffic area- $S_{3}$ ) including at site $S_{4}$ (Tirupati city area) and both category plants are highly beneficial for mitigation and minimization of air pollution load and also for landscaping purpose of the area as they tend to serve as barriers and act as sink for air pollutants. The species of moderate category i.e. Catharanthus roseus (OP-23) may be recommended for plantation due to its aesthetic value for landscaping. Sensitive plant species on the other hand act as bioindicators of air pollution and thus can be planted in order to check the environmental health from time to time. High pollution levels can lead to deforestation in long run and thus this kind of study helps in understanding the plants susceptibility and resistance to pollution loads.

\section{References}

Akilan, M. and Nandhakumar, S. 2016. Air pollution tolerance index of selected plants in industrial and urban areas of Vellore district. Agricultural Science Digest. 36(1): 66-68.

Badamasi, H. 2017. Biomonitoring of air pollution using plants. Journal of Environmental Sciences. 2: 27-39.

Bates, I.S, Waldren, R.P. and Tarc, I.D. 1973. Rapid determination of free proline for water stress studies. Plant Soil. 39: 207.

Bhattacharya, T, Chakraborty, B, Kagathara, M. and Thakur, B. 2012. Ambient air quality and the air pollution tolerance indices of some common plant species of Anand city, Gujarat, India. Report and Opinion. 4(9): 7-15.

Bora, M. and Joshi, N. 2014. A study on variation in biochemical aspects of different tree species with tolerance and performance index. The Bioscan. 9(1): 59-63.

Chandawat, D.K, Verma, P.U. and Solanki, H.A. 2011. Air pollution tolerance index (APTI) of tree species at cross roads of Ahmedabad city. Life Sciences Leaflets. 20: 935-943.

Chandawat, D.K, Verma, P.U, Solanki, H.A. and Patel, Y.M. 2014. Role of total phenol in the resistance mechanism of plants against air pollution. Biolife. 2(2):586-592.

Chaudhary, P. and Banerjee, D. 2009. Biomonitoring of air quality in the industrial town of Asansol using the air pollution tolerance index approach. Research Journal of Chemistry and 
Environment. 13(1): 45-51.

Das, S. and Prasad, P. 2010. Seasonal variation in air pollution tolerance indices and selection of plant species for industrial areas of Rourkela. Indian Journal of Environmental Protection. 30(12): 978-88.

Deepalakshmi, A.P. Ramakrishnaiah, H, Ramachandra, Y.L. and Radhika, R.N. 2013. Roadside plants as bio-indicators of urban air pollution. Journal of Environmental Science, Toxicology and Food Technology. 3: 10-14.

Gharge, S. and Menon, G.S. 2012. Air pollution tolerance index (APTI) ofcertain herbs from the site around Ambernath MIDC. Asian Journal of Experimental Biological Sciences. 3(3):543-547.

Ghassanen, R.A, Morsy, A.A. and Saleh, H.A. 2016. Leaf dust accumulation and air pollution tolerance indices of three plant species exposed to urban particulate matter pollution from a fertilizer factory. International Journal of Plant Research. 29:146-51.

Govindaraju, M, Kumar, G.R.S, Muthukumaran, V.R. and Visvanathan, P. 2012. Identification and evaluation of air-pollution-tolerant plants around lignite-based thermal power station for greenbelt development. Environmental Science and Pollution Research. 19: 1210-1223.

Jyothi, J.S. and Jaya, D.S. 2010. Evaluation of air pollution tolerance index of selected plant species along roadsides in Thiruvanthapuram, Kerala. Journal of Environment and Biology. 31: 379386.

Kaur, M. and Nagpal, A.K. 2017. Evaluation of Air Pollution Tolerance Index and Anticipated Performance Index of plants and their application in development of green space along the urban areas. Environmental Science and Pollution Research. 24: 1888118895.

Kousar, H, Kumar, N.D, Pavithra, K. and Patel, A.M. 2014. Analysis of biochemical parameters as tolerance index of some chosen plant species of Bhadravathi town. International Journal of Environmental Science. 3: 11-16.

Lichtenthaler, H. K. and Buschmann, C. 1987. Chlorophylls and carotenoids: Pigments of photosynthetic biomembranes. Met. Enzy. 148: 350382.

Madan, S. and Chauhan, S. 2015. Air pollution tolerance index and anticipated performance index of selected plant species in Haridwar city, India. Report and Opinion. 7:32-37.

Meerabai, G, Ramana, V.C. and Rasheed, M. 2012. Effect of industrial pollutants on physiology of Cajanus cajan (L.) fabaceae. International Journal of Environmental Sciences. 2(4): 18921894.

Mohsenpour, P, Amjad, L. and Yahyaabadi, S. 2015. The effect of environmental pollutions on phytochemical parameters and antioxidant activity of Quince, Apple, and Mulberry fruits. Journal of Biodiversity and Environmental Sciences. 6(1):282-289.

Noor, M.J, Sultana, S, Fatima, S, Ahmad, M, Zafar, M, Sarfraz, M, Balkhyour, M.A, Safi, S.Z. and Ashraf, M.A. 2015. Estimation of Anticipated Performance Index and Air Pollution Tolerance Index of vegetation around the marble industrial areas of Potwar region: bioindicators of plant pollution response. Environmental Geochemistry and Health. 37:441-455.

Nwadinigwe, A.O. 2014. Air pollution tolerance indices of some plants around Ama industrial complex in Enugu State, Nigeria. African Journal 
of Biotechnology.13(11): 1231-1236.

Prajapati, S.K. and Tripathi, B.D. 2008. Anticipated performance index of some tree species considered for green belt development in and around an urban area: a case study of Varanasi city. Indian Journal of Environmental Management. 88:1343-1349.

Prasad, B.J. and Rao, D.N. 1982. Relative sensitivity of a leguminous and a cereal crop to sulphur dioxide pollution. Environmental Pollution. 29:57-70.

Rai, P.K, Lalith, L.S, Panda, Chutia, B.M. and Singh, M.M. 2013. Comparative assessment of air pollution tolerance index (APTI) in the industrial area (Rourkela) and non industrial area (Aizawl), India: An eco-management approach. African Journal of Environmental Science and Technology. 7(10): 944-948.

Ranganna, S. 1977. Hand book of analysis and quality control for fruit and vegetable products. Tata Mc. Graw Hill publishing Co. Ltd., New Delhi. 29-31.

Singh A. 1977. Practical Plant Physiology. Kalyari Publishers. New Delhi.

Singleton, V.I, Joseph, A. and Rossi. 1965. Colorimetry of total phenolics with phosphomolybdic- phosphotungstic acid reagents. American Journal of Enology and Viticulture. 16:144-158.

Soni, A, Aseri, G.K.and Jain, N. 2017. Impact of air pollution caused by mining and marble dust on foliar sensitivity through biochemical changes. International Journal of Engineering Research and Technology. 5(12): 8083.

Tsega, Y.C. and Deviprasad, A.G. 2014. Variation in air pollution tolerance index and anticipated performance index of roadside plants in Mysore, India Journal of Environmental Biology. 35: 185-190.

\section{How to cite this article:}

Amrutha Pavani, N., M. Raja Naik, R. Nagaraju, G. Srinivasa Rao, V. V. Padmaja and Srinivasulu, B. 2020. Response of Ornamental Plants, Study Sites and their Interaction to Various Pollutants in Respect of APTI, API, Physiological and Biochemical Attributes. Int.J.Curr.Microbiol.App.Sci. 9(12): 37-49. doi: https://doi.org/10.20546/ijcmas.2020.912.007 